\title{
Dental follicle mesenchymal stem cells regulate responses in sepsis
}

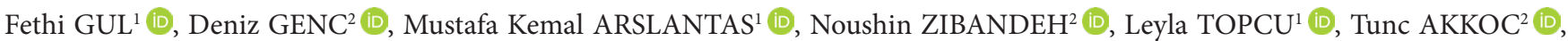 \\ Ismail CINEL ${ }^{1}$ (D) \\ 1 Department of Anesthesiology and Reanimation, School of Medicine, Marmara University Training and Research Hospital, Istanbul, Turkey. \\ 2 Department of Pediatric Allergy and Immunology, School of Medicine, Marmara University Training and Research Hospital, Istanbul, Turkey.
}

Corresponding Author: Ismail CINEL

E-mail: cinelismail@yahoo.com

Submitted: 06.11.2019 Accepted: 18.12.2019

\begin{abstract}
Objective: Sepsis-induced immune alterations are associated with secondary infections and increased risk of death. The use of mesenchymal stem cells (MSCs) has been described as a novel therapeutic strategy. We evaluated the immunomodulatory effects of human dental follicle (DF-MSCs) on lymphocytes of sepsis and septic shock patients.

Materials and Methods: Peripheral blood mononuclear cells (PBMCs) were isolated from venous blood samples of sepsis, septic shock and healthy subjects. PBMCs were co-cultured in the presence and absence of DF-MSCs with or without interferon-gamma (IFN- $\gamma$ ) for 72 hours. CD4+CD25+FoxP3+regulatory T (Treg) cell frequency, lymphocyte proliferation, cytokine levels and apoptosis were evaluated via flow cytometry.

Results: DF-MSCs significantly suppressed proliferation of lymphocytes in sepsis group compared to septic shock group ( $\mathrm{p}<0.005$ ). DF-MSCs remarkably increased Treg ratio in sepsis compared to control group $(\mathrm{p}<0.05)$. Reduction of lymphocyte apoptosis in cocultures of DF-MSCs and PBMC was significant in both sepsis and septic shock groups. IFN- $\gamma$ stimulation of DF-MSCs ameliorated shift in the T-cell subsets from Th2 to Th1 phenotype in septic shock.

Conclusion: Our findings revealed that DF-MSCs have immunoregulatory effects both in sepsis and septic shock, by reducing interleukin-4 (IL-4) and increasing IFN- $\gamma$ levels. This immunoreactivity regulation may open new therapeutic approaches for septic shock patients.

Keywords: Sepsis, Septic shock, Dental follicle mesenchymal stem cells, Immunomodulation
\end{abstract}

\section{INTRODUCTION}

Several international societies convened an international task force to review the definitions of sepsis, septic shock, and related conditions recently and sepsis was defined as a life-threatening organ dysfunction caused by a dysregulated host response to infection by the task force [1]. It is one of the main causes of morbidity and mortality in critically ill patients despite advances in our understanding of the pathogenesis [2]. Mortality has declined modestly with improved ventilation strategies and fluid management protocols but remains high according to data from the Surviving Sepsis Campaign [3,4].

Increasing evidence suggests that both innate immune and inflammatory responses are involved in the pathophysiology of sepsis [5]. Pro-inflammatory and anti-inflammatory processes begin promptly after sepsis initiation and in a short time hyperinflammatory phase occurs, and that follows with the anti-inflammatory phase of sepsis [6]. Immunosuppression has a critical role in sepsis, and this immune response occurs by the cytokine production that leads to end-organ damage. Potential mechanisms of immunosuppression in sepsis are the deviation of response from inflammatory to anti-inflammatory phase, anergy and loss of T-helper cells (Th1) and immunity [7].

Mesenchymal stem cells (MSCs) are multipotent adult progenitor cells that have the capacity to self-renew and differentiate into various cell lineages and they can be easily isolated from various tissues, including bone marrow, umbilical cord, and adipose tissues $[8,9]$. They have immunomodulatory and regenerative effects that make them attractive therapeutic candidates for a potential treatment option for diseases and immune disorders $[10,11]$. It is considered that MSCs can give rise to multilineage progenitor cells and play an active role in immunoregulatory processes by responding to the inflammatory challenge with the production of anti-inflammatory factors [12]. In recent

How to cite this article: Gul F, Genc D, Arslantas MK, Zibandeh N, Topcu L, Akkoc T, Cinel I. Dental follicle mesenchymal stem cells regulate responses in sepsis. Marmara Med J 2020;33: 7-16 doi: 10.5472/marumj.681996 
years, many studies have proven that immunomodulation with stem cells is an important mechanism underlying the benefits of strengthening repair of damaged tissue and can therefore provide a therapeutic option for sepsis [13,14]. Dental follicle mesenchymal stem cells (DF-MSCs) have more advantages than other stem cells due to their doubling time, immunomodulation effects and tissue repair capabilities. The rapid proliferative capacity together with the immunoregulatory characteristics of dental tissue-derived MSCs may prompt future studies aimed at using these cells in the treatment or prevention of inflammatory diseases [15]. Our previous studies showed that DF-MSCs regulated Th1 and Th2 responses by increasing $\mathrm{T}$ regulatory (Treg) cell population and kept the effectory $\mathrm{T}$ lymphocytes in silent stage and blocked the antigen presentation by downregulating the costimulatory molecules $[16,17]$.

In the present study, the immunomodulatory properties of DFMSCs were investigated by evaluating lymphocyte proliferation, apoptosis, induction of regulatory Treg cells and cytokine profile in sepsis and septic shock in vitro. The mechanisms have been studied by determining the changes in inflammation-associated cytokine profiles and assessing the immunomodulatory effect of interferon-gamma (IFN- $\gamma$ ) on DF-MSCs in sepsis.

\section{MATERIALS and METHODS}

\section{Selection of patients and study groups}

Patients between the ages of 18 and 65 years with the diagnosis of sepsis or septic shock were included in the study. According to the 2001 International Sepsis Definitions Conference Report, patients divided into two groups as sepsis (Group I: mean age 29.8 $\pm 7.2, \mathrm{n}=10$ ) and septic shock (Group II: mean age $49.3 \pm 14.6, \mathrm{n}=10$ ). Written informed consent was obtained from all patients. Peripheral blood mononuclear cells (PBMCs) were isolated from venous blood samples of Group I, Group II and

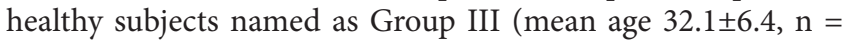
10).

Exclusion criteria were those who used anti-inflammatory drugs, corticosteroids, antibiotics and who received chemotherapy, radiotherapy or immunotherapy in the last six months. Patients were included in the study according to the 2001 International Sepsis Definitions Conference Report. Septic patients were defined as a systemic response to infection with the presence of some degree of organ dysfunction. Septic shock patients were who had sepsis-induced hypotension despite adequate fluid resuscitation along with the presence of perfusion abnormalities that may include but are not limited to lactic acidosis or oliguria.

\section{Isolation, characterization and differentiation potential of DF-MSCs}

Isolation: Dental follicle tissues were obtained from 4 volunteer adult donors aged between 20 and 25 years undergoing thirdmolar extraction without abscess. Tissue samples were taken from individuals who were not diagnosed with a genetic disease or did not receive chemotherapy, radiotherapy or immunotherapy. DFMSCs were isolated and cultured as described previously [18].
Briefly, dental follicles were seperately cut into $0.5-1 \mathrm{~mm}$ pieces and enzimatically digested with $3 \mathrm{mg} / \mathrm{mL}$ collagenase type I in phosphate buffer solution (PBS - Sigma-Aldrich,USA) at $37^{\circ} \mathrm{C}$. After incubation period, tissue samples were filtered through 70 micron naylon filter and obtained cell suspension was washed twice with Dulbecco's modified eagle medium (DMEM) (Gibco, Thermofisher Scientific, USA) supplemented with 10\% fetal bovine serum (FBS) (Sigma-Aldrich, USA) and centrifuged at $1200 \mathrm{rpm}$ for 5 minutes. Cells were then suspended in DMEM supplemented with $10 \%$ FBS and $1 \%$ penicillin/streptomycin (hereafter refered as cDMEM). Each $5 \times 10^{5}$ cells were cultured in $\mathrm{T} 25$ flask at $37^{\circ} \mathrm{C}$ and $5 \mathrm{CO}_{2} \%$ humidified incubator until reaching $80-90 \%$ confluency and subjected to the next passage. Cells were used at the third passage.

Characterization: For cell surface antigen phenotyping, third passage cells were stained with fluorescein and phycoerythrincoupled antibodies and analyzed with FACSCalibur (BD). Cells were analyzed for positive markers; CD29 (APC), CD73 (PE), CD90 (FITC), CD106 (PerCp) for MSCs and negative markers; CD14 (APC), CD34 (FITC), CD45 (FITC) and HLA-DR (PE) were used. All antibodies were provided from BD Biosciences, USA.

Differentiation potential of DFSCs: To evaluate the multipotency of DF-MSCs, we stimulated the third passage cells for osteogenic, chondrogenic and adipogenic differentiation. For osteogenic differentiation, third passage cells were treated with osteogenic medium for three weeks with medium changes twice weekly. The osteogenic medium contains basal medium supplemented with $0.1 \mu \mathrm{M}$ dexamethasone (SigmaAldrich, USA), $10 \mathrm{mM}$ - glycerol phosphate (Sigma-Aldrich, USA), and $0.2 \mathrm{mM}$ ascorbic acid (Sigma-Aldrich, USA). The chondrogenic differentiation medium was supplemented with $0.1 \mu \mathrm{M}$ dexamethasone, $100 \mu \mathrm{g} / \mathrm{ml}$ sodium pyruvate (SigmaAldrich, USA), $40 \mu \mathrm{g} / \mathrm{mL}$ proline (Sigma-Aldrich, USA), 6.25 $\mu \mathrm{g} / \mathrm{mL}$ insulin, $6.25 \mu \mathrm{g} / \mathrm{mL}$ transferrin, $6.25 \mathrm{ng} / \mathrm{mL}$ selenious acid, $1.25 \mathrm{mg} / \mathrm{mL}$ bovine serum albumin (BSA - Abcam, UK), and $5.35 \mathrm{mg} / \mathrm{mL}$ linoleic acid) in the basal medium (Mesencult $\mathrm{tm})$. To induce adipogenic differentiation, third passage cells were treated with the adipogenic medium for three weeks. The medium was changed twice weekly. The adipogenic medium contains basal medium (Mesencult ${ }^{\text {tm }}$, Stemcell Technologies, CA) supplemented with $0.5 \mathrm{mM} 3$-isobutyl-1-methylxanthine (Sigma-Aldrich, USA), $1 \mathrm{mM}$ hydrocortisone (Sigma-Aldrich, USA), $0.1 \mathrm{mM}$ indomethacin (Sigma-Aldrich, USA), and $10 \%$ FBS (Sigma-Aldrich, USA).

\section{Stimulation of DF-MSCs with IFN- $\gamma$}

DF-MSCs in the third passage were inoculated in 48 well plates $5 \times 10^{4}$ cells in $0,5 \mathrm{ml}$ of DMEM supplemented with $10 \%$ FBS and $1 \%$ penicillin/streptomycin (Gibco, Thermo Ffisher, USA) (hereby refered as complete DMEM) in each well. DF-MSCs were incubated for 24 hours to be confluent to the plate. After the incubation period, the medium was removed from wells and changed with stimulation media (IFN $-\gamma 0,5 \mu \mathrm{g} / \mathrm{ml}$, complete DMEM) and incubated for additional 24 hours. 


\section{Collection of venous blood samples and isolation of PBMCs}

Blood samples of patients were obtained from Marmara University Training and Research Hospital, Department of Anesthesiology and Reanimation under approved guidelines of Marmara University School of Medicine Ethical Board (70737436-050.06.04). Heparinized blood was collected with central venous catheter. The blood was transported and processed in the research laboratory within 30 minutes. PBMCs were isolated by ficoll-paque density gradient solution and resuspended in cell culture media (RPMI) 1640 supplemented with $10 \%$ FBS, $1 \%$ penicillin/streptomycin.

\section{DF-MSCs and PBMCs co-cultures}

Culture conditions: PBMCs were cultured in the presence and absence of DF-MSCs (IFN- $\gamma^{+}$or IFN- $\gamma^{-}$) with the ratio of $1: 10$ (MSCs: PBMC) in $0.5 \mathrm{ml}$ of RPMI 1640 supplemented with $10 \%$ FBS and 1\% penicillin/streptomycin in each well of 48 well plates and lymphocytes were stimulated with anti-CD3 and anti-CD28 (eBioscience, Thermo Fisher Scientific, USA) purified antibodies. PBMCs in the presence and absence of DFMSCs (IFN- $\gamma^{+}$or IFN- $\gamma^{-}$) were cultured for 72 hours to evaluate lymphocyte proliferation, apoptosis, and regulatory $\mathrm{T}$ cell ratio in cultured PBMCs.

Lymphocyte Proliferation Assay: T cell proliferation ratio was measured by the cell surface staining with carboxyfluorescein diacetate succinimidyl ester (CFSE)(e-Bioscience, Thermo Fisher, USA). Lymphocytes $\left(5 \times 10^{5} /\right.$ well $)$ cultured in 48 well plates were stained with $1.8 \mu \mathrm{M}$ CFSE and incubated for 6 minutes. After incubation period cells were washed with $10 \%$ FBS in phosphate-buffered saline (PBS). After 72 hours of the culture period, cells were analyzed by BD FACSCalibur flow cytometer (BD FACSCalibur Biosciences, USA). In order to analyze $\mathrm{T}$ lymphocyte proliferation the CD3+ gated cells were subjected to CFSE signaling.

Lymphocyte Apoptosis Assay: T cell apoptosis ratio was analyzed in PBMC cultures with and without DF-MSCs (IFN- $\gamma^{+}$ or IFN- $\gamma^{-}$) after 72 hours of culturing period. T-cell apoptosis assays were performed in 48-well round-bottom plates in a total volume of $0.5 \mathrm{~mL}$ RPMI 1640 medium with 10\% FBS and $1 \%$ penicillin/streptomycin. After 72 hours of the culture period, cells were washed twice with PBS and stained with anti-CD3 (PE), Annexin V (Fluorescein isothiocyanate; FITC) and Propidium Iodide (Peridinin-Chlorophyll-protein; PerCp) (Ebioscience) and analyzed by BD FACSCalibur flow cytometer. Analysis was performed by gating CD3+ cells for Annexin V and propium iodite (PI). All antibodies were provided from $\mathrm{BD}$ Biosciences, USA.

Analysis of Treg cells. After incubation period cells were washed with PBS and remaining cell pellet were evaluated for Treg cells. Treg cell ratios were analyzed by intracellular staining of FoxP3 (PerCp) and surface staining with anti-CD4 (FITC) and anti-CD25 (Phycoerythrin; PE). All staining protocols were performed according to the manufacturer's instructions. Treg cells were identified as $\mathrm{CD}^{+}$and $\mathrm{CD} 25^{+}$that labeled with FoxP3. In the analysis, CD25 gated cells were analyzed for CD4+ and FoxP3+ cells. All samples were analyzed by BD FACSCalibur flow cytometer. All antibodies were provided from BD Biosciences, USA.

Analysis of Cytokine Levels: After 3 days of the culture period, the supernatants were collected and quantified for the cytokines IL-4, IL-6, IL-10, TNF-a and IFN- $\gamma(\mathrm{pg} / \mathrm{mL})$. The cytokines were analyzed via flow cytometry using a BD cytometric bead array (CBA) human Th1/Th2/Th17 Kit (BD Biosciences, USA) according to the manufacturer's instructions.

\section{Statistical Analysis}

For statistical analysis, SPSS 21 software was used (SPSS, SPSS Inc., Chicago). Analysis of variance (ANOVA) for repeated measures, Student's t-test, and Fisher's exact test were used, as appropriate. Sub - group analyses were performed using ANOVA with Fisher's post hoc test. All data were checked for normal distribution (Kolmogorov - Smirnov test). Nonnormally distributed data were log transformed. Results refer to the intention-to-treat population and are reported as mean \pm standard deviation (S.D.), if not indicated otherwise. $p$ value less than 0.05 was considered significant.

\section{RESULTS}

Patients with sepsis $(n=10)$, septic shock $(n=10)$ and healthy individuals as control $(n=10)$ were included in this study. Inclusion and exclusion criteria for study subjects was shown in Figure 1. Peripheral blood samples were collected, and PBMCs were isolated and cultured with and without DF-MSCs $\left(\right.$ IFN- $\gamma^{+}$or IFN- $\gamma^{-}$) with the stimulation of anti-CD3/anti-CD28 of PBMCs for 72 hours to evaluate immunomodulatory effect of DFMSCs on lymphocytes and inflammation parameters. DF-MSCs were either stimulated with IFN- $\gamma$ or non-stimulated for the observation of the immunomodulatory effect of inflammatory cytokine IFN- $\gamma$ on DF-MSCs. At the end of the culture period lymphocyte proliferation, apoptosis and regulatory $\mathrm{T}$ cell ratios and cytokine levels were analyzed by flow cytometry.

\section{Isolation, Characterization, Multipotency of DF-MSCs}

DF-MSCs exhibited a fibroblast-like and spindle-shaped morphology in the first few days of incubation. DF-MSCs proliferated in 3 days and formed fibroblast-like colonies (Figure 2A) in the primary culture in their first passage. Third passage cells were analyzed by flow cytometry and with surface markers for positive staining for CD29, CD73, CD90, CD106 and were negative for CD14, CD34, CD45, and HLA-DR (Figure 2B). The DFSCs differentiated well into osteocytes, chondrocytes, and adipocytes. The osteogenic differentiation of DF-MSCs was evaluated with Alizarin red staining for calcium phosphate deposits. DF-MSCs showed the morphology of osteoblasts and osteocytes and calcium phosphate deposits were stained in the intracellular matrix. The in vitro adipogenic differentiation capability was assessed by culturing the cells in an adipogenic stimulated medium, and at the end of the culture period stained with Oil Red O. Intracellular lipid droplets were observed in these cells. The chondrogenic differentiation capability was observed 
in vitro during a twenty-one-day culture period in chondrogenic medium, and cell differentiation into chondrocytes was observed with Alcian blue staining. Proteoglycans were observed in the culture (Figure 2C).

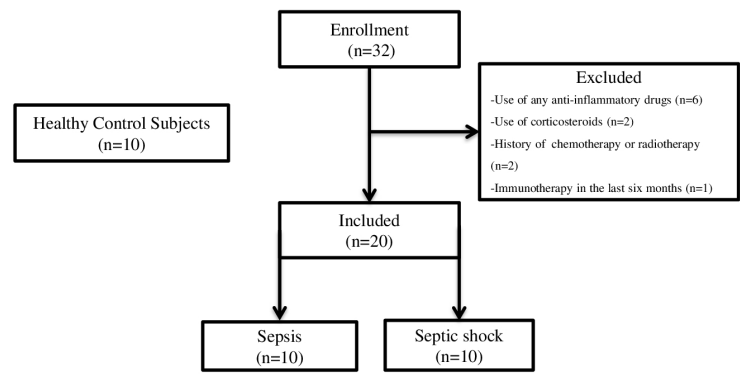

Figure 1. Flow diagram of the 20 patients with sepsis and septic shock who were identified among the 32 patients enrolled. (Healthy control subjects, $\mathrm{n}=10$ )
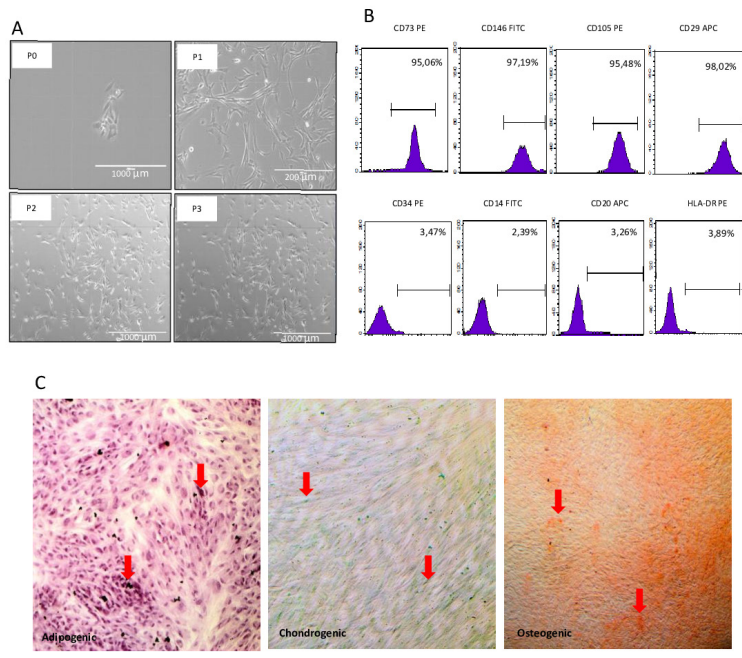

Figure 2. Isolation, characterization and differentiation of DF-MSCs. A) DF-MSCs formed fibroblast-like colonies at passage 0 (P0), P1, P2 and $\mathrm{P} 3$ on the fourth day of inoculation. B) DF-MSCs were analyzed in the third passage via flow cytometry and expressed positive markers of CD29, CD73, CD105 and CD146, and lack the expression of negative markers of CD14, CD34, CD45 and HLA-DR. C) DF-MSCs were differentiated into osteoblasts, chondrocytes and adipocytes after stimulation. Cells were stained with alizarin red, oil red $\mathrm{O}$ and alcian blue at the end of the culture period for osteogenic, adipogenic and chondrogenic differentiation of DF-MSCs respectively. Red arrows show calcium deposits in osteogenic differentiation.

\section{DF-MSCs suppressed lymphocyte proliferation in sepsis and septic shock patients}

PBMCs were isolated from peripheral blood and cultured for 72 hours. They were labeled with CFSE and incubated with and without DF-MSCs (IFN- $\gamma^{+}$or IFN- $\gamma^{-}$), analyzed by BD FACSCalibur flow cytometry via CFSE labeling for proliferation. Based on the hypothesis that the initiation of activity of MSCs during inflammatory responses such as antigen presentation and T-cell proliferation, we treated DFMSCs with regulatory protein IFN- $\gamma$ to increase its ability in the initiation of DF-MSCs' suppressive activity. The proliferation ratio of PBMC cultures without DF-MSCs varied between the groups. Lymphocyte proliferation ratio was significantly high in anti-CD3/CD28 stimulated cultures without DF-MSCs in sepsis group when compared with septic shock and control groups $(\mathrm{p}<0.05)$. DF-MSCs, both IFN- $\gamma$ prestimulated and unstimulated significantly suppressed lymphocyte proliferation in sepsis $(p<0.001$ and $p<0.005)$, septic shock $(p<0.005$ and $\mathrm{p}<0.05)$, and control groups $(\mathrm{p}<0.01$ and $\mathrm{p}<0.01)$ compared to PBMC cultures alone (Figure 3 ).
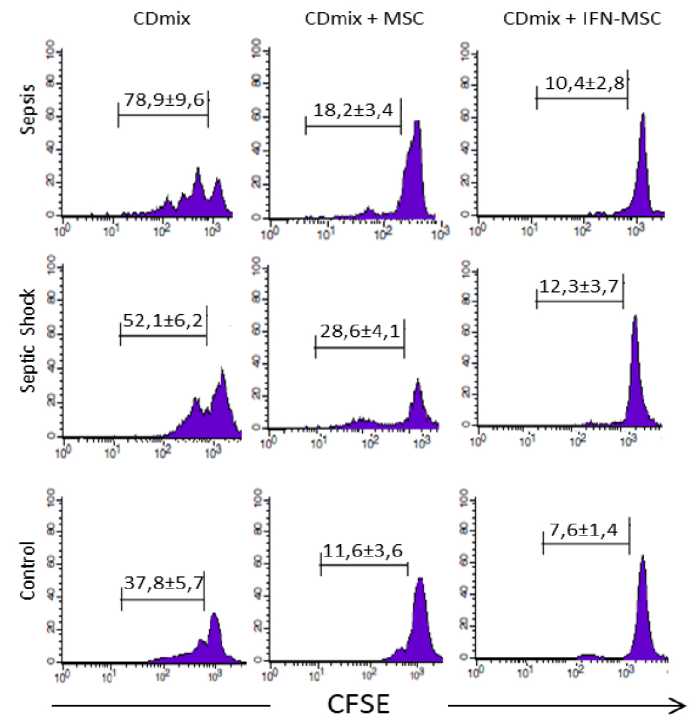

Figure 3. Lymphocyte proliferation. CFSE labeled cells were analyzed via flow cytometry and counted for proliferative responses to stimulants. Lymphocyte proliferation ratio with anti-CD3/anti-CD28 (CDmix) stimulation in $\mathrm{PBMC}$ of sepsis patients was significantly high compared to septic shock and healthy subjects $(\mathrm{p}<0.05)$. DF-MSCs significantly decreased proliferative response in all groups but the reduction in sepsis patients was noteworthy $(\mathrm{p}<0.005)$, and IFN- $\gamma$ prestimulated DF-MSCs further decreased proliferation rates of lymphocytes in sepsis patients $(\mathrm{p}<0.001)$.

\section{DF-MSCs increased the lymphocyte survival in sepsis and septic shock patients}

PBMCs were analyzed after 72 hours of the culture period for $\mathrm{T}$ lymphocyte apoptosis. Lymphocytes were incubated with and without DF-MSCs (IFN- $\gamma^{+}$or IFN- $\gamma^{-}$), and apoptosis was analyzed via BD FACSCalibur flow cytometry by labeling with anti-CD3, Annexin V and PI. The viability of lymphocyte was significantly decreased in sepsis $(\mathrm{p}<0.005)$ and septic shock groups $(\mathrm{p}<0.001)$ in the absence of DF-MSCs compared to control group. DF-MSCs with and without IFN- $\gamma$ stimulation significantly increased the viability of lymphocyte by decreasing 
apoptosis compared to PBMC cultures without DF-MSCs in sepsis and septic shock groups $(\mathrm{p}<0.01$, and $\mathrm{p}<0.001$, respectively). IFN $-\gamma$ prestimulation of DF-MSCs further decreased the lymphocyte apoptosis in all groups, but it was significant in sepsis group compared to un-stimulated DFMSCs in the co-cultures $(\mathrm{p}<0.01)$. Apoptotic profiles differ within groups. In CDmix stimulated PBMC cultures sepsis (51.7 \pm 4.1$)$ and septic shock patients' $(59.3 \pm 5.6)$ lymphocytes were mostly undergone necrosis, while $4.9 \pm 2.3$ of lymphocytes showed necrosis in healthy subjects. DF-MSCs with or without

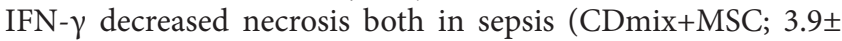
0.7, CDmix+IFN-MSC; $4.1 \pm 0.9)$ and septic shock (12.4 \pm 3.1$)$ patients' lymphocytes. Early apoptosis was increased in CDmix cultures of healthy individuals $(11.5 \pm 1.8)$ without DF-MSCs, despite this, cell survival ratio of lymphocytes was high in control group (75.3 \pm 4.9$)$ compared to sepsis and septic shock patients. IFN- $\gamma$ stimulation of DF-MSCs decreased either early and late apoptosis in healthy individuals or necrosis in septic and septic shock patients. Moreover, lymphocyte survival significantly increased in sepsis, septic shock and tend to increase in healthy subjects in the presence of DF-MSCs (Figure 4).
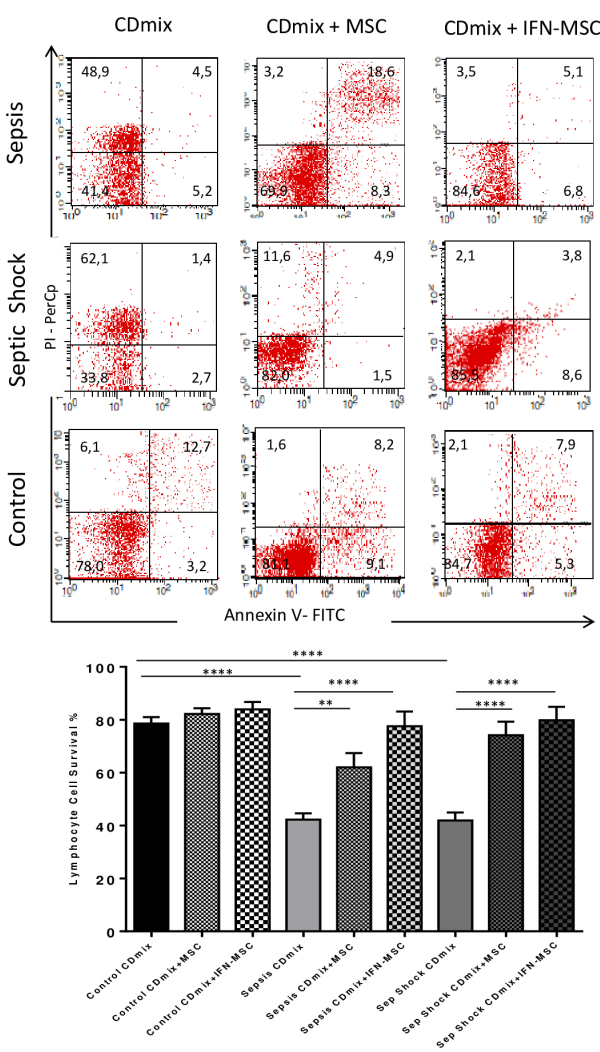

Figure 4. Lymphocyte apoptosis. Apoptotic rates of lymphocytes in PBMC cultures were quantified via flow cytometry. Cell survival ratio of lymphocytes was significantly low in sepsis $(\mathrm{p}<0.001)$ and septic shock patinets $(\mathrm{p}<0.001)$ compared to healthy subjects. Unstimulated DF-MSCs increased cell survival and decreased apoptotic rates of lymphocytes in sepsis $(\mathrm{p}<0.01)$ and septic shock patients $(\mathrm{p}<0.001)$ significantly compared to PBMC cultures alone, and IFN- $\gamma$ prestimulated DF-MSCs notably increased lymphocyte survival in sepsis group when compared with un-stimulated DF-MSCs $(\mathrm{p}<0.01)$ and PBMC cultures alone $(\mathrm{p}<0.001) .{ }^{* *} ; \mathrm{p}<0.01,{ }^{* * * *} ; \mathrm{p}<0.001$.

\section{DF-MSCs enhanced the number of $\mathrm{CD}^{+} \mathrm{CD}^{+} 5^{+} \mathrm{FoxP}^{+}$Treg cells}

We studied the effects of DF-MSCs on the Treg cell frequency. Seventy-two hours after culture, PBMC were collected and analyzed for CD4 and CD25 with cell surface markers, and intracellular staining with the FoxP3 antibody. CD4 ${ }^{+} \mathrm{FoxP} 3^{+}$Treg cell population differed between the groups. Treg cell ratio was lower in the sepsis group compared to septic shock and control groups in anti-CD2/anti-CD3/anti-CD28 stimulated PBMC without DF-MSCs. Un-stimulated DF-MSCs significantly increased Treg ratio in sepsis $(\mathrm{p}<0.001)$ and control group $(\mathrm{p}<0.05)$ compared to PBMC cultures alone, whereas there was no significant change observed in septic shock patients in cocultures $(p>0.05)$. IFN- $\gamma$ prestimulation of DF-MSCs further increased Treg ratio in sepsis group compared to PBMC cultures alone $(\mathrm{p}<0.001)$, but there was no significant difference between co-cultures of un-stimulated DF-MSCs. In septic shock group, Treg ratio was significantly higher in PBMC cultures without stem cells compared to sepsis and control groups $(\mathrm{p}<0.05)$. DFMSCs tend to increase the Treg ratio in septic shock group, but it was not significant (Figure 5).

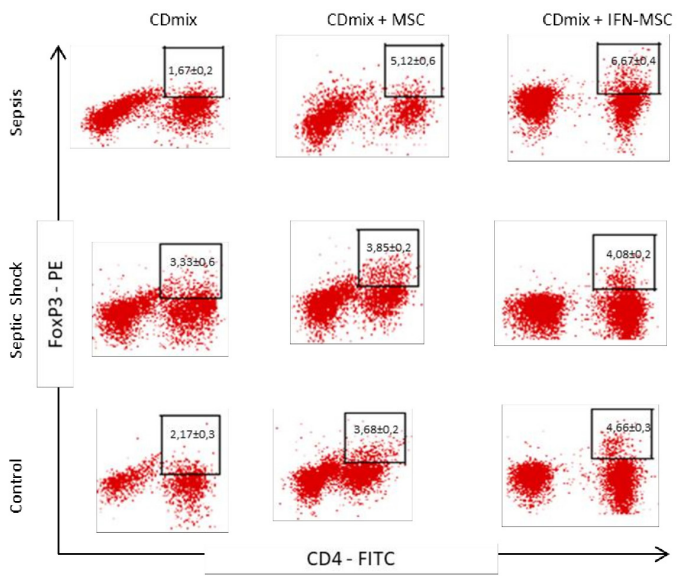

Figure 5. $\mathrm{CD}^{+} \mathrm{FoxP}^{+} \mathrm{T}$ regulatory cell ratio. Un-stimulated DFMSCs significantly increased Treg ratio in sepsis $(\mathrm{p}<0.001)$ and control group (0.05) compared to PBMC cultures alone, whereas there was no significant change observed in septic shock patients in cocultures $(\mathrm{p}>0.05)$. IFN- $\gamma$ prestimulation of DF-MSCs further increased Treg ratio in sepsis group compared to PBMC cultures alone $(\mathrm{p}<0.001)$. Treg ratio was significantly higher in PBMC cultures without stem cells in septic shock group, compared to sepsis and control groups $(\mathrm{p}<0.05)$. DF-MSCs tend to increase the Treg ratio in septic shock group, and it was not significant. 


\section{DF-MSCs regulated the proinflammatory cytokine levels in sepsis and septic shock patients}

We analyzed the proinflammatory and anti-inflammatory cytokine levels in culture supernatants to evaluate the immunoregulatory effects of DF-MSCs in sepsis and septic shock patients. The supernatants were collected after 72 hours of culture of PBMC in the presence and absence of DF-MSCs $\left(\mathrm{IFN}-\gamma^{+}\right.$or IFN- $\left.\gamma^{-}\right)$. Further, we analyzed IL-4, IL-6, IL-10, IFN- $\gamma$, and TNF-a cytokine levels after culture period.

The proinflammatory cytokine levels (IL-6 and TNF-a) were significantly higher in sepsis group PBMC when cultured without stem cells compared to septic shock and control groups (IL-6; sepsis-septic shock $<0.01, \mathrm{p}_{\text {seppis-control }}<0.001$ and TNF-a; sepsis-septic shock $<0.01$, $\left.\mathrm{p}_{\text {sepsis-control }}<0.001\right)$. DF-MSCs significantly decreased IL-6 and TNF-a levels in sepsis and septic shock groups compared to PBMC cultures without stem cells (sepsis; $\mathrm{p}<0.001, \mathrm{p}<0.001$ and septic shock; $\mathrm{p}<0.01, \mathrm{p}<0.01$, respectively). However, IFN- $\gamma$ prestimulated DF-MSCs significantly decreased IL-6 levels compared to unstimulated DF-MSCs in septic shock group $(\mathrm{p}<0.01)$ while there was no difference in sepsis group. IFN- $\gamma$ level was significantly lower in PBMC cultures of septic shock group compared to sepsis patients $(\mathrm{p}<0.05)$. Unstimulated and IFN- $\gamma$ prestimulated DF-MSCs significantly increased IFN- $\gamma$ levels in the co-cultures of septic shock patients $(\mathrm{p}<0.01)$, whereas, it was significantly decreased in sepsis group and control group compared to PBMC cultures without DF-MSCs $(\mathrm{p}<0.001)$.

IL-4 level was higher in sepsis group when compared with control group, but it was not significant. DF-MSCs tend to decrease IL-4 levels in the co-cultures in sepsis group. Additionally, the IL-4 level was significantly higher in PBMC cultures of septic shock group compared to sepsis and control groups $\left(\mathrm{p}_{\text {septic shock- }}\right.$ control $\left.<0.001, \mathrm{p}_{\text {septic shock-sepsis }}<0.005\right)$. DF-MSCs decreased IL-4 levels in septic shock group significantly when compared with PBMC cultures without stem cells $(\mathrm{p}<0.005)$. IFN- $\gamma$ prestimulation of DF-MSCs further decreased IL-4 levels compared to CDmix cultures of PBMC in septic shock group $(\mathrm{p}<0.001)$. We further analyzed $\mathrm{Th}_{1}(\mathrm{IFN}-\gamma) / \mathrm{Th}_{2}$ (IL-4) ratio to understand the effect of MSCs in immune deviation of sepsis and septic shock patients. Our results revealed that Th2 immunity was dominant in septic shock patients while DF-MSCs deviated it to Th1 immunity which was important for survival (Table I and Table II).
Table I. Change in the IFN- $\gamma$ levels in culture groups with DF$\operatorname{MSCs}( \pm \mathrm{IFN}-\gamma)$

\begin{tabular}{|l|l|l|l|l|}
\hline Group & Culture Condition & $\begin{array}{l}\text { IFN- } \gamma \text { Level } \\
(\mathrm{pg} / \mathrm{mL}) \\
\text { Average value }\end{array}$ & $\begin{array}{l}\text { Change } \\
\text { in the } \\
\text { cytokine } \\
\text { level }\end{array}$ & $\begin{array}{l}\text { Change in the } \\
\text { cytokine level } \\
\text { in percentage } \\
(\%)\end{array}$ \\
\hline Control & CDmix & 647.52 & & \\
\hline & CDmix+MSC & 302.05 & $\downarrow \downarrow$ & 53 \\
\hline & CDmix+IFN- $\gamma$ MSC & 198.27 & $\downarrow \downarrow \downarrow$ & 69 \\
\hline Sepsis & CDmix & 524.12 & & \\
\hline & CDmix+MSC & 156.40 & $\downarrow \downarrow$ & 70 \\
\hline & CDmix+IFN- $\gamma$ MSC & 179.23 & $\downarrow \downarrow \downarrow$ & 65 \\
\hline $\begin{array}{l}\text { Septic } \\
\text { Shock }\end{array}$ & CDmix & 47.84 & & \\
\hline & CDmix+MSC & 163.35 & $\uparrow \uparrow$ & 241 \\
\hline & CDmix+IFN- $\gamma$ MSC & 217.10 & $\uparrow \uparrow \uparrow$ & 353 \\
\hline
\end{tabular}

IFN- $\gamma$; Interferron gamma, MSC; Mesenchymal Stem Cell, IFN- $\gamma$ MSC; Interferron gamma stimulated mesenchymal stem cell

Table II. Change in the IL-4 levels in the culture groups with DF-MSCs $( \pm \mathrm{IFN}-\gamma)$

\begin{tabular}{|l|l|l|l|l|}
\hline Group & Culture Condition & $\begin{array}{l}\text { IL-4 level (pg/ } \\
\mathrm{mL}) \\
\text { Average value }\end{array}$ & $\begin{array}{l}\text { Change } \\
\text { in the } \\
\text { cytokine } \\
\text { level }\end{array}$ & $\begin{array}{l}\text { Change in the } \\
\text { cytokine level } \\
\text { in percentage } \\
(\%)\end{array}$ \\
\hline Control & CDmix & 1.86 & & \\
\hline & CDmix+MSC & 1.07 & $\downarrow \downarrow$ & 42 \\
\hline & CDmix+IFN- $\gamma$ MSC & 0.92 & $\downarrow \downarrow \downarrow$ & 50 \\
\hline Sepsis & CDmix & 3.27 & & \\
\hline & CDmix+MSC & 3.19 & $\downarrow \downarrow$ & 0,02 \\
\hline & CDmix+IFN- $\gamma$ MSC & 0.89 & $\downarrow \downarrow$ & 72 \\
\hline $\begin{array}{l}\text { Septic } \\
\text { Shock }\end{array}$ & CDmix & 21.06 & & \\
\hline & CDmix+MSC & 4.18 & $\downarrow \downarrow$ & 80 \\
\hline & CDmix + IFN- $\gamma$ MSC & 2.86 & $\downarrow \downarrow \downarrow$ & 86 \\
\hline
\end{tabular}

IL; Interleukin, MSC; Mesenchymal Stem Cell, IFN- $\gamma$ MSC; Interferron gamma stimulated mesenchymal stem cell

IL-10 level was significantly higher in PBMC cultures of septic shock group compared to sepsis and control groups ( $\mathrm{p}_{\text {septic shock- }}$ control $\left.<0.05, \mathrm{p}_{\text {septic shock-sepsis }}<0.01\right)$. DF-MSCs significantly increased IL-10 levels in sepsis group compared to PBMC cultures without stem cells $(\mathrm{p}<0.001)$ and further increased by pretreatment of DF-MSCs with IFN- $\gamma$. There was no significant difference between PBMC cultures and DF-MSCs cocultures in septic shock group in IL-10 levels (Figure 6). 

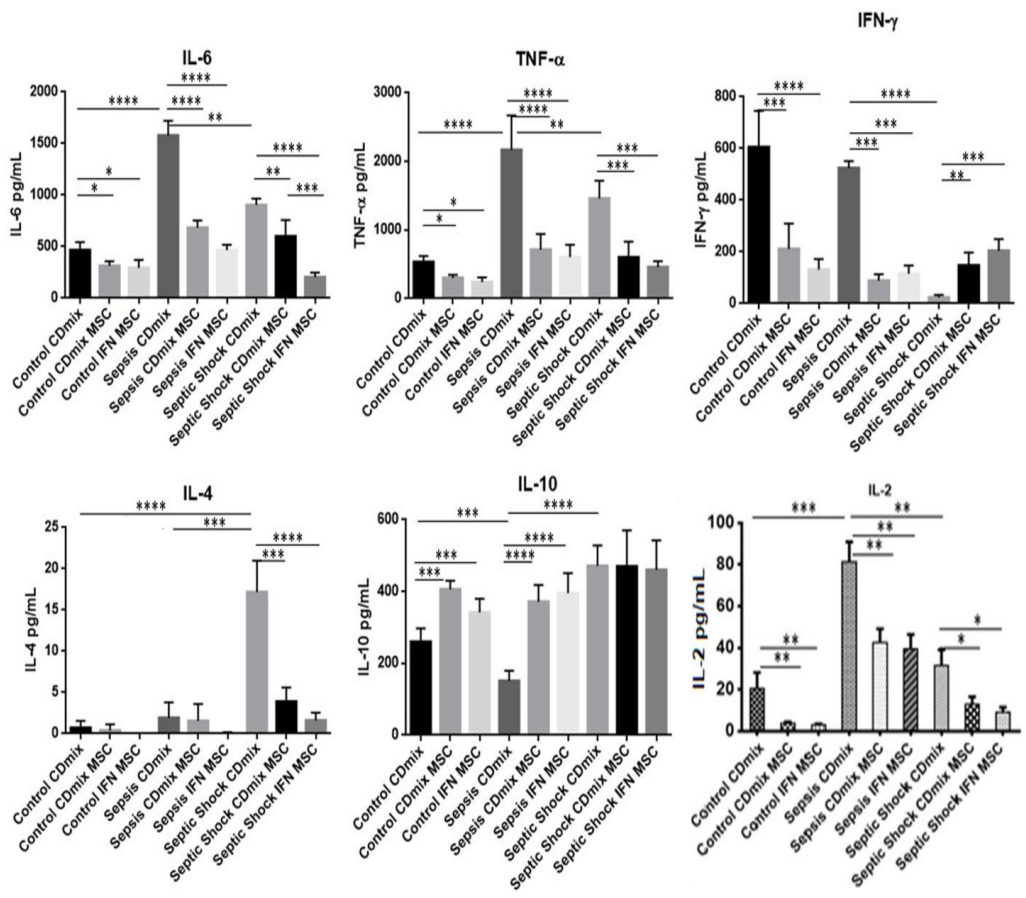

Figure 6. Proinflammatory and anti-inflammatory cytokine levels. Cytokine levels in culture supernatants were analyzed after 72 hours via flow cytometry and values were given in $\mathrm{pg} / \mathrm{mL}$. Statistical values of; IL-6, TNF- $\alpha$, IFN- $\gamma$, IL-4, IL-10, IL-2 levels were shown. ${ }^{*}: \mathrm{p}<0.05,{ }^{* *}$ : $<<0.01,{ }^{* * *}$ : $\mathrm{p}<0.005$ and $^{* * *}: \mathrm{p}<0.001$

\section{DISCUSSION}

Sepsis is characterized by overwhelming systemic activation of the immune response due to the release of various molecules from invading microbial pathogens or damaged host tissue [19]. The pathophysiology of sepsis associates interplay between pro - and anti-inflammatory responses. In the proinflammatory response phase cytokines such as tumor necrosis factor (TNF)-a, IL-1b, IL-6, and IFN- $\gamma$ are produced which stimulate the effector functions of mononuclear cells $[20,21]$. In the current study, we evaluated the modulatory effects of DF-MSCs as an alternative source of MSCs, on lymphocytes isolated from sepsis and septic shock patients and compared those with healthy individuals. We demonstrated that DF-MSCs can modulate TNF-a and IL-6 levels in PBMC supernatants and regulate the proliferative response of T cells in sepsis patients by enhancing Treg frequency, while reducing Treg cell ratio and IL-10 levels and shifting Th2 cells in favour of Th1 cells in septic shock patients.

Sepsis leads to changes in immunity and results in immune paralysis. Anti-inflammatory stage of the host response to sepsis, characterized by apoptosis of T-cell, paralysis of macrophage and shift in the T-cell subsets from Th1 to the Th2 phenotype is mediated by Treg cells [9]. Immunodepression also occurs by decrease of IFN- $\gamma$ levels and the increase of IL-10 and IL-4 levels during this T cell phenotype shift [22-24].

DF-MSCs are multipotent cells with short doubling time thus can reach the required number of cells in early passages [25].
MSCs have been recently reported to have an anti-inflammatory potential in experimental models of sepsis [26]. Recently, the immunosuppressive effect of dental mesenchymal stem cells was studied and reported that DF-MSCs have a strong immunomodulatory impact on lymphocytes [18]. However, it is still unclear whether DF-MSCs regulate sepsis-induced inflammatory responses of lymphocytes.

In this study, we demonstrated that DF-MSCs can reduce the proliferative response of $\mathrm{T}$ lymphocytes in PBMC of sepsis patients, by increasing $\mathrm{CD} 4^{+} \mathrm{CD} 25^{+} \mathrm{FoxP} 3^{+}$Treg cell frequency and increasing IL-10 levels in vitro. Although, it was not noticeable in sepsis patients, the proliferation of T lymphocytes was lowered with DF-MSCs in septic shock patients. The regulatory effect of DFSCs on proliferation of lymphocytes of sepsis and septic shock patients was further augmented with the stimulation of IFN- $\gamma$. But, elevated Treg cell ratio and IL10 levels did not change notably in septic shock patients' PBMC in the presence of DF-MSCs. We evaluated the difference in the decrease of lymphocyte proliferation in the presence of unstimulated or IFN- $\gamma$ stimulated DF-MSCs in sepsis and septic shock patients together with T lymphocyte apoptosis, Treg cell frequency and cytokine level.

In this study, we investigated the apoptosis and viability ratio of lymphocytes in sepsis and septic shock patients and evaluated the effect of DF-MSCs on cell viability. Lymphocyte apoptosis was higher in sepsis and septic shock patients compared to 
healthy individuals in PBMC cultures. DF-MSCs significantly decreased apoptosis and increased viability of lymphocytes in sepsis and septic shock patients, which demonstrated the antiapoptotic effect of DF-MSCs. These data indicated that the decrease in proliferation of $\mathrm{T}$ lymphocytes was not caused by apoptosis. In previous studies, it was shown that MSCs had an anti-apoptotic effect on activated lymphocytes by contactdependent mechanisms $[27,28]$, and modulated the function of several immune cells which released inflammatory mediators (IFN $\gamma, \operatorname{IL} 1 \beta$ and TNF $\alpha)[23,29,30]$. In this regard, our results were comparable with the previous studies.

Anti-inflammatory responses with IL-10 expression by Treg cells in sepsis is a critical parameter. One of the studies showed that increase in the IL-10 level in the early phase of sepsis resulted with the improvement of the disease, but harmful, depending on the time of intervention [31]. In our study, we analyzed IL-10 levels in PBMC cultures of sepsis and septic shock patients and observed that level of this cytokine was significantly increased in septic shock compared to sepsis and control groups. Results showed that DF-MSCs did not change IL-10 levels in septic shock in comparison with PBMC cultures but significantly upregulated IL-10 secretion in sepsis group compared to PBMC cultures without stem cells. In other words, DF-MSCs modulated aggregated inflammatory response in sepsis whereas no change occurred in septic shock patients which is a critical point for the immunosuppression.

IL-2 is the key mediator of T lymphocyte proliferation and promotes the expansion of $\mathrm{T}$ lymphocytes [32]. In our study, IL-2 levels were high in sepsis patients which indicated increased proliferative responses and induced activated lymphocytes for clonal expansion. DF-MSCs decreased IL-2 levels in co-cultures of PBMC of sepsis patients remarkably. These data indicated that DF-MSCs regulated the lymphocyte proliferation by increasing the number of Treg cells and decreasing IL-2 levels. IFN- $\gamma$ prestimulation of DF-MSCs enhanced Treg cell ratio and further decreased IL-2 levels in sepsis group, whereas there was no significant change in Treg cell ratio and IL-2 level in septic shock group and IL-2 level in healthy individuals. These data suggested that DF-MSCs may have a limited effect on effector functions of inactivated lymphocytes but have the major inhibitory effect on activated lymphocytes.

Our data indicated that immune cell profiles played a critical role in the host response to sepsis. Following the early phase, the immune cell phenotype shifted towards immune-suppression status. While, sepsis patients in early phase had Th1 (T helper 1) cell mediated immune responses with the secretion of proinflammatory cytokines (IFN- $\gamma$, TNF-a, IL-6, IL-12), septic shock patients had predominantly Th2 cell mediated responses with the secretion of anti-inflammatory cytokines (IL-4, IL10) which showed the suppressive phase [33-35]. IL-4 is a cytokine with variable immunoregulatory functions, and has an important action in the differentiation of naive $\mathrm{T}$ lymphocyte towards Th2 type cells and anti-inflammatory mechanism suddenly occurs in this period of sepsis which could lead to aggressive immune destructions [36,37]. IL-4 causes an enhanced release of further IL- 4 and other anti-inflammatory cytokines and results with immunosuppression [33]. A recent study demonstrated the increase in the mortality ratio was correlated with high expression of IL-4 [38]. In this study, we investigated IL-4 levels in PBMCs of sepsis and septic shock patients. The elevated level of IL-4 in septic shock patients was significant compared to sepsis and healthy individuals, which indicated that Th2 mediated anti-inflammatory response was dominant in septic shock patients. DF-MSCs reduced IL-4 levels in septic shock patients' co-cultures while no remarkable change was observed in sepsis and healthy subjects. This reduction in the cytokine level in septic shock patients provides hope of returning to anti-inflammatory phase of septic shock by DFMSCs. On the contrary, some of the studies demonstrated that bone marrow or adipose tissue derived MSCs enhanced IL-4 production of $\mathrm{T}$ lymphocytes $[39,40]$ in sepsis murine models. The reason for this difference in the results may be the use of different cell sources (bone marrow, adipose tissues, dental tissues) and the lymphocyte profile of sepsis or septic shock.

In a study, lymphocyte anergy or unresponsiveness occurred resulting in immune system declining, which caused the immune system susceptibility to bacterial and viral infections \{41]. Clinical studies signed that the duration of sepsis-induced immune alterations was associated with increased risk of death [42]. Some biomarkers are important in disease progression for sepsis. Recent studies demonstrated that increased IFN- $\gamma$ level resulted in improved patient outcomes with the restoration of immune cell functions in sepsis and septic shock [33]. In our study, we evaluated the effect of DF-MSCs on IFN- $\gamma$ levels in sepsis and septic shock. IFN- $\gamma$ level was significantly lower in septic shock patients compared to sepsis patients and healthy volunteers in PBMC cultures without stem cells. DF-MSCs increased IFN- $\gamma$ levels in PBMC of septic shock patients, whereas downregulated in sepsis and healthy individuals. Also, IFN- $\gamma$ prestimulation of DF-MSCs further increased IFN- $\gamma$ levels in septic shock patients. This may be an evidence of selective immunoregulatory effect of DF-MSCs on distinctly activated T lymphocytes.

The current study demonstrates that DF-MSCs can downregulate the inflammatory responses in sepsis patients and can modulate Th2 mediated responses in favor of Th1 cells in septic shock patients. This data shows variable regulatory functions of DFMSCs in sepsis in response to cytokine profile and inflammatory conditions, and further in vivo studies are required to confirm the regulatory role of DF-MSCs in sepsis.

\section{Limitations}

A power analysis was not performed to determine sample size because we did not have preliminary data of the immunomodulatory properties of DF-MSCs in sepsis and septic shock in vitro to perform a power analysis. Thus, this study must be considered an exploratory pilot study rather than a formal test of a hypothesis. 


\section{Conclusion}

In this study, we demonstrated that IFN- $\gamma$ stimulation of DFMSCs had regulatory effects on inflammatory responses in sepsis, and reduced lymphocyte apoptosis in septic shock. DFMSCs may be further investigated to use as a novel treatment strategy by the mean of restoring immune system homeostasis for sepsis and septic shock in vivo studies, and also the way of administration and dose responses can be determined.

\section{Ethical Committee Approval}

This study was approved by Marmara University, Clinical Research Ethics Committee.

\section{Conflict of Interest}

The authors declare that they have no conflict of interest

Author Contributions: Concept and Design - FG, IC; Supervision - IC; Resources - IC, TA; Materials - DG, NZ, TA; Data Collection and Processing - FG, DG, MKA; Analysis and Interpretation - FG, DG, NZ, MKA; Literature Search - FG, DG, LT; Writing Manuscript - FG, DG, IC; Critical Review IC, FG, TA.

\section{REFERENCES}

[1] Singer M, Deutschman CS, Seymour CW, et al. The Third International Consensus Definitions for Sepsis and Septic Shock (Sepsis-3). JAMA 2016; 315:801-10. doi:10.1001/ jama.2016.0287

[2] Angus DC, Linde-Zwirble WT, Lidicker J, Clermont G, Carcillo J, Pinsky MR. Epidemiology of severe sepsis in the United States: analysis of incidence, outcome, and associated costs of care. Crit Care Med 2001; 29:1303-10. doi: 10.1097/00003.246.200107000-00002

[3] Kumar G, Kumar N, Taneja A, et al. Nationwide trends of severe sepsis in the 21st century (2000-2007). Chest 2011; 140:1223-31. doi: 10.1378/chest.11-0352.

[4] Kaukonen KM, Bailey M, Suzuki S, Pilcher D, Bellomo R. Mortality related to severe sepsis and septic shock among critically ill patients in Australia and New Zealand, 2000-2012. JAMA 2014;311:1308-16. doi: 10.1001/jama.2014.2637.

[5] Kurosawa DJS, Osuchowski MF, Valentine C, Kurosawa S, Remick DG. The pathogenesis of sepsis. Annu Rev Pathol 2011; 6: 19-48. doi:10.1146/annurev-pathol-011.110.130327.

[6] Sundar KM, Sires M. Sepsis-induced immunosuppression: Implications for secondary infections and complications. Indian J Crit Care Med 2013; 17: 162-69. doi: 10.4103/09725229.117054 .

[7] Hotchkiss RS, Monneret G, Payen D. Immunosuppression in sepsis: a novel understanding of the disorder and a new therapeutic approach. Lancet Infect Dis 2013; 13: 260-8. doi: 10.1016/S1473-3099(13)70001-X.

[8] Pittenger MF, Mackay AM, Beck SC, et al. Multilineage potential of adult human mesenchymal stem cells. Science 1999;284:143-7. doi: 10.1126/science.284.5411.143.
[9] Huang GTJ, Gronthos S, Shi S. Mesenchymal stem cells derived from dental tissues vs. those from other sources. J Dent Res 2009; 88: 792-806. doi: 10.1177/002.203.4509340867.

[10] Le Blanc K, Frassoni F, Ball L, et al. Developmental Committee of the European Group for Blood and Marrow Transplantation. Mesenchymal stem cells for treatment of steroid-resistant, severe, acute graft-versus-host disease: a phase II study. Lancet 2008; 371(9624): 1579-86. doi: 10.1016/ S0140-6736(08)60690-X.

[11] Kovach TK, Dighe AS, Lobo PI, Cui Q. Interactions between MSCs and Immune Cells: Implications for Bone Healing. J Immunol Res 2015; 2015:752510. doi: 10.1155/2015/752510.

[12] Zhang Q, Shi S, Liu Y, et al. Mesenchymal stem cells derived from human gingiva are capable of immunomodulatory functions and ameliorate inflammation-related tissue destruction in experimental colitis. J Immunol 2009; 15:183:7787-98. doi: 10.4049/jimmunol.0902318.

[13] Lombardo E, Poll T, DelaRosa O, Dalemans W. Mesenchymal stem cells as a therapeutic tool to treat sepsis. World J Stem Cells 2015; 7: 368-79. doi: 10.4252/wjsc.v7.i2.368

[14] Boomer JS, To K, Chang KC, et al. Immunosuppression in patients who die of sepsis and multiple organ failure. JAMA 2011; 306: 2594-605. doi: 10.1001/jama.2011.1829.

[15] Pierdomenico L, Bonsi L, Calvitti M, et al. Multipotent mesenchymal stem cells with immunosuppressive activity can be easily isolated from dental pulp. Transplantation 2005; 80 : 836-42. doi: 10.1097/01.tp.000.017.3794.72151.88

[16] Genç D, Zibandeh N, Nain E, et al. Dental follicle mesenchymal stem cells downregulate th2 mediated immune response in asthmatic patients mononuclear cells. Clin Exp Allergy 2018; 48:663-78. doi: 10.1111/cea.13126

[17] Genç D, Zibandeh N, Nain E, et al. IFN- $\gamma$ stimulation of dental follicle mesenchymal stem cells modulates immune response of CD4+T lymphocytes in Der p1+ asthmatic patients in vitro. Allergol Immunopathol (Madr) 2019;S0301-0546(19)300047. doi: 10.1016/j.aller.2018.12.005

[18] Yildirim S, Zibandeh N, Genc D, Ozcan EM, Goker K, Akkoc T. The comparison of the immunologic properties of stem cells isolated from human exfoliated deciduous teeth, dental pulp, and dental follicles. Stem Cells Int 2016; 2016:4682875. doi: $10.1155 / 2016 / 4682875$

[19] Scott MJ, Godshall CJ, Cheadle WG. Jaks, STATs, cytokines, and sepsis. Clin Diagn Lab Immunol 2002; 9: 1153-9. doi: 10.1128/CDLI.9.6.1153-1159.2002

[20] Rittirsch D, Flierl MA, Ward PA. Harmful molecular mechanisms in sepsis. Nat Rev Immunol 2008; 8: 776-87. doi: 10.1038/nri2402.

[21] Chong DL, Sriskandan S. Pro-inflammatory mechanisms in sepsis. Contrib Microbiol 2011; 17: 86-107. doi: $10.1159 / 000324022$.

[22] Leentjens J, Kox M, van der Hoeven JG, Netea MG, Pickkers P. Immunotherapy for the adjunctive treatment of sepsis: from immunosuppression to immunostimulation. Time for a paradigm change? Am J Respir Crit Care Med 2013; 187: 1287-93. doi: 10.1164/rccm.201.301.0036CP. 
[23] Nalos M, Santner-Nanan B, Parnell G, Tang B, McLean AS, Nanan R. Immune effects of interferon gamma in persistent staphylococcal sepsis. Am J Respir Crit Care Med 2012; 185:110-2. doi: 10.1164/ajrccm.185.1.110.

[24] Sivanathan KN, Gronthos S, Rojas-Canales D, Thierry B, Coates PT. Interferon-gamma modification of mesenchymal stem cells: implications of autologous and allogeneic mesenchymal stem cell therapy in allotransplantation. Stem Cell Rev 2014; 10:351-75. doi: 10.1007/s12015.014.9495-2.

[25] Morsczeck C, Völlner F, Saugspier M, et al. Comparison of human dental follicle cells (DFCs) and stem cells from human exfoliated deciduous teeth (SHED) after neural differentiation in vitro. Clinical Oral Investig 2010; 14: 43340. doi: 10.1007/s00784.009.0310-4.

[26] Rey EG, Anderson P, González MA, Rico L, Büscher D, Delgado M. Human adult stem cells derived from adipose tissue protect against experimental colitis and sepsis. Gut 2009; 58: 929-39. doi: 10.1136/gut.2008.168534.

[27] Bernardo ME, Fibbe WE. Mesenchymal stromal cells: sensors and switchers of inflammation. Cell Stem Cell 2013; 13:392402. doi: 10.1016/j.stem.2013.09.006.

[28] Akiyama K, Chen C, Wang D, et al. Mesenchymal-stemcell-induced immunoregulation involves FAS-ligand-/FASmediated T cell apoptosis. Cell Stem Cell 2012; 10:544-55. doi: 10.1016/j.stem.2012.03.007.

[29] Krampera M, Cosmi L, Angeli R, et al. Role for interferongamma in the immunomodulatory activity of human bone marrow mesenchymal stem cells. Stem Cells 2006; 24:386-98. doi: 10.1634/stemcells.2005-0008

[30] Prasanna SJ, Gopalakrishnan D, Shankar SR, Vasandan AB. Pro-inflammatory cytokines, IFN gamma and TNF alpha, influence immune properties of human bone marrow and Wharton jelly mesenchymal stem cells differentially. PLoS One 2010; 5:e9016. doi: 10.1371/journal.pone.0009016

[31] Reinhart K, Bauer M, Riedemann NC, Hartog CS. New approaches to sepsis: molecular diagnostics and biomarkers. Clin Microbiol Rev 2012; 25: 609-34. doi: 10.1128/ CMR.00016-12.
[32] Osinalde N, Mitxelena J, Sánchez-Quiles V, et al. Nuclear phosphoproteomic screen uncovers ACLY as mediator of IL2-induced proliferation of CD4+ T lymphocytes. Mol Cell Proteomics 2016; 15:2076-92. doi: 10.1074/mcp.M115.057158.

[33] Surbatovic M, Popovic N, Vojvodic D, et al. Cytokine profile in severe gram-positive and gram-negative abdominal sepsis. Sci Rep 2015; 5:11355. doi: 10.1038/srep11355

[34] Lang JD, Matute-Bello G. Lymphocytes, apoptosis and sepsis: making the jump from mice to humans. Crit Care 2009; 13:109. doi: 10.1186/cc7144.

[35] Rimmelé T, Payen D, Cantaluppi V, et al. Immune cell phenotype and function in sepsis. Shock 2016; 45:282-91. doi: 10.1097/SHK.000.000.0000000495.

[36] Wherry EJ. T cell exhaustion. Nat Immunol 2011; 12: 492-9. doi: 10.1038/ni.2035.

[37] Hotchkiss RS, Monneret G, Payen D. Sepsis-induced immunosuppression: from cellular dysfunctions to immunotherapy. Nat Rev Immunol 2013; 13: 862-74. doi: 10.1038/nri3552.

[38] Ward PA. Immunosuppression in Sepsis. JAMA 2011; 306:2618-9. doi: 10.1001/jama.2011.1831.

[39] Schlosser K, Wang JP, Dos Santos C, et al. Effects of mesenchymal stem cell treatment on systemic cytokine levels in a phase 1 dose escalation safety trial of septic shock patients. Crit Care Med 2019;47:918-25. doi: 10.1097/ CCM.000.000.0000003657.

[40] Wang Y, Tan L, Jin J, et al. Non-cultured dermal-derived mesenchymal cells attenuate sepsis induced by cecal ligation and puncture in mice. Sci Rep 2015; 5: 16973. doi: 10.1038/ srep 16973

[41] Bone RC. Immunologic dissonance: a continuing evolution in our understanding of the systemic inflammatory response syndrome (SIRS) and the multiple organ dysfunction syndrome (MODS). Ann Intern Med 1996; 125:680-7. doi: 10.7326/0003-4819-125-8-199610.150.00009

[42] Latifi SQ, O’Riordan MA, Levine AD. Interleukin-10 controls the onset of irreversible septic shock. Infect Immun 2002; 70 : 4441-6. doi: 10.1128/iai.70.8.4441-4446.2002 